\title{
Lessons of the month: Not just morning sickness
}

\author{
Author: Chin Voon Tong ${ }^{\mathrm{A}}$
}

\begin{abstract}
A 34-year-old woman (gravida 4; para 3) at 17 weeks of pregnancy presented with abnormal behaviour for 3 weeks associated with difficulties in walking. She had been admitted 2 months prior with hyperemesis gravidarum and was also diagnosed with thyrotoxicosis. Vomiting and poor oral intake persisted after discharge. She was euthyroid otherwise. Clinical examination revealed a dehydrated and confused patient. She had nystagmus and ataxic gate. Magnetic resonance imaging of the brain showed symmetrical signal changes of posteromedial part of thalamus. A diagnosis of Wernicke's encephalopathy (WE) was made and intravenous thiamine was given leading to gradual improvement of symptoms. Her thyroid function test normalised without any treatment. WE can occur in severe hyperemesis gravidarum. Prompt recognition of WE and replacement with thiamine is important to prevent neurological sequelae and mortality. Gestational transient thyrotoxicosis, which is self-limiting, is more prominent in patients with hyperemesis gravidarum and requires only symptomatic treatment.
\end{abstract}

KEYWORDS: Wernicke's encephalopathy, hyperemesis gravidarum, gestational transient thyrotoxicosis

DOI: 10.7861/clinmed.2020-0151

\section{Case presentation}

A 34-year-old woman (gravida 4; para 3) at 17 weeks of pregnancy was referred to our hospital with abnormal behaviour for 3 weeks associated with difficulties in walking. She was talking irrelevantly at home and had visual hallucinations. Two months earlier, she was admitted to the referring hospital with hyperemesis gravidarum. Biochemically she had hyperthyroidism with elevated free thyroxine (T4) of $32.23 \mathrm{pmol} / \mathrm{L}$ and suppressed thyroidstimulating hormone (TSH) of $0.008 \mathrm{mU} / \mathrm{L}$. She had been given propylthiouracil since then but was not able to tolerate the medication. After discharge, she continued to have persistent vomiting, up to ten times a day with poor oral intake. She did not have any past history of thyroid disorders nor family history of similar illness. On examination she was dehydrated, lethargic and confused. She had horizontal nystagmus and ataxic gait. Cranial nerves were intact and there were no other neurological deficits.

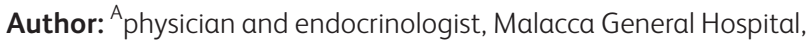
Malacca, Malaysia
Babinski's reflexes were down-going bilaterally. She did not have any thyroid orbitopathy or goitre. Clinically she was euthyroid.

Blood investigations showed normochromic normocytic anaemia with haemoglobin of $82 \mathrm{~g} / \mathrm{L}$, white blood count of $11.4 \times 10^{9} / \mathrm{L}$ and platelet count of $393 \times 10^{9} / \mathrm{L}$. Renal profile showed hypokalaemia with potassium of $3 \mathrm{mmol} / \mathrm{L}$. Free T4 was $30.1 \mathrm{pmol} / \mathrm{L}$ with TSH of $0.01 \mathrm{mU} / \mathrm{L}$. TSH receptor antibody was negative. Urine ketone was $2+$. The referring doctor had arranged for magnetic resonance imaging (MRI) of the brain without contrast which showed symmetrical signal changes of the posteromedial part of thalamus (Fig 1).

A diagnosis of Wernicke's encephalopathy was made. She was given intravenous thiamine (vitamin $B_{1}$ ) $200 \mathrm{mg}$ three times a day for 5 days followed by $200 \mathrm{mg}$ twice a day for 3 days and subsequently switched to maintenance oral form $100 \mathrm{mg}$ once daily. Intravenous saline with potassium supplements were also initiated along with anti-emetics. Electrolytes were monitored closely to avoid precipitating central pontine myelinolysis (CPM). Her abnormal thyroid function test was attributed to gestational thyrotoxicosis and hyperemesis gravidarum. Propylthiouracil was stopped. She was referred to our rehabilitation team for active physiotherapy. Her condition improved gradually, and she was able to walk on her own. During clinic review 1 month after being discharged, she still had residual giddiness but was able to eat and mobilise on her own. Her thyroid function test had normalised without any medications.

\section{Discussion}

Wernicke's encephalopathy (WE) is an acute but potentially reversible neurological syndrome caused by thiamine deficiency. Thiamine plays an important role as a cofactor for several enzymes which are pertinent in energy metabolism. ${ }^{1}$ In conditions which lead to higher metabolic demand and glucose intake, thiamine requirements will be increased. Mechanism of neuronal damage caused by thiamine deficiency include mitochondrial damage, cell apoptosis and oxidative stress. ${ }^{2}$ Although often associated with chronic alcoholism, WE can occur in conditions that lead to poor nutrition or increased metabolic requirements. Lesser known causes of WE include severe hyperemesis gravidarum, anorexia nervosa and post-bariatric surgery complications. In hyperemesis gravidarum, WE occurs as a result of excessive vomiting, poor intake and increased metabolic demand. ${ }^{3}$ In hindsight, our patient presented with classic triad of WE which include confusion, ataxia and ophthalmoplegia. Only about one-third of patients with WE present classically. ${ }^{4}$ There is no specific laboratory blood test to diagnose WE. Thiamine level, even if available, does not reflect 

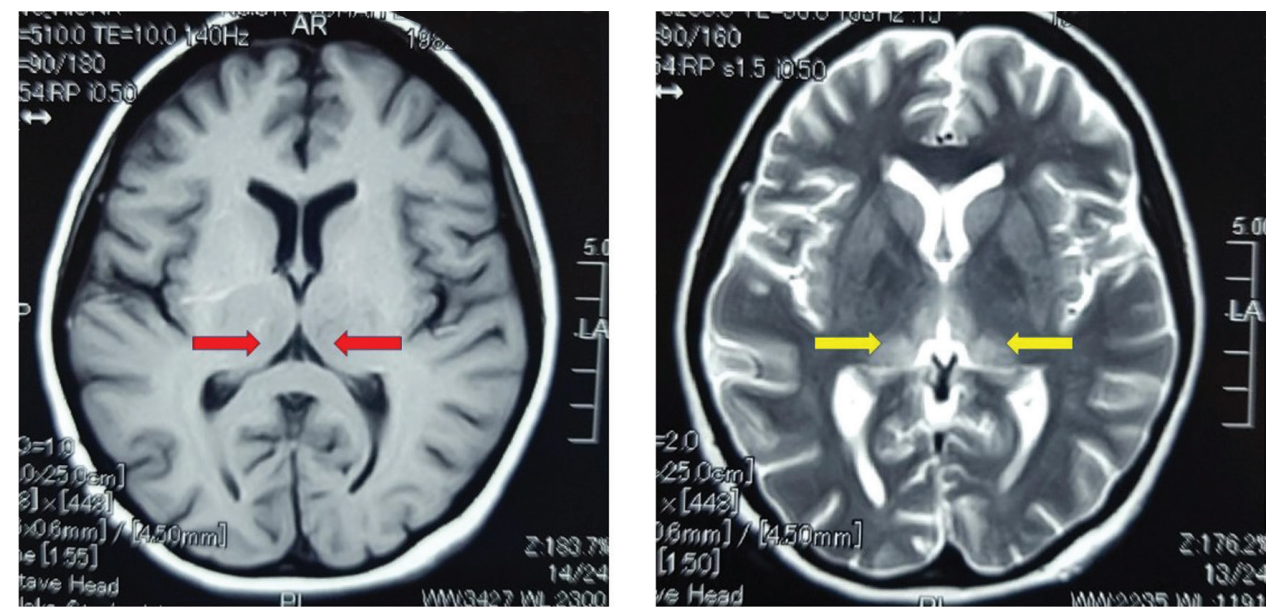

Fig 1. Magnetic resonance imaging of the brain (non-contrasted) showing symmetrical signal changes of the posteromedial part of the thalamus which is hypointense on T1 (red arrows) and hyperintense on T2 (Yellow arrows)

cerebral thiamine level. ${ }^{5}$ Prompt recognition of WE is important as early treatment with intravenous thiamine, which is safe, simple and efficacious, can prevent further complications including death. Glucose loading prior to initiation of thiamine can also potentially precipitate or worsen WE. Imaging studies are usually not required to diagnose WE but may help exclude other diagnoses. MRI findings that have been described include symmetrical changes in the thalami, mamillary bodies, tectal plate and periaqueductal area. $^{6}$

Biochemically, our patient had hyperthyroidism which was due to gestational transient thyrotoxicosis. This phenomenon is caused by molecular mimicry between human chorionic gonadotrophin (hCG) and TSH. ${ }^{7}$ hCG, which peaks at 10-12 weeks gestation and is higher in patients with hyperemesis gravidarum, has mild thyroid-stimulating activity. ${ }^{8}$ Absence of symptoms, goiter and TSH receptor antibodies help distinguish gestational transient thyrotoxicosis from thyrotoxicosis in pregnancy which would require treatment with anti-thyroid medications. Clinching a proper diagnosis is important to avoid initiation of unnecessary therapies.

\section{Key points}

$>$ WE can occur in severe hyperemesis gravidarum. Prompt recognition of WE and replacement with thiamine is important to prevent neurological sequelae and mortality.

> Gestational transient thyrotoxicosis, which is self-limiting, is more prominent in patients with hyperemesis gravidarum and requires only symptomatic treatment.

\section{References}

1 Victor M, Adams RA, Collins GH. The Wernicke-Korsakoff syndrome and related disorders due to alcoholism and malnutrition. Philadelphia: FA Davis, 1989.

2 Martin PR, Singleton CK, Hiller-Sturmhöfel S. The role of thiamine deficiency in alcoholic brain disease. Alcohol Res Health 2003;27:134.

3 Kantor S, Prakash S, Chandwani J, Gokhale A, Sarma K, Albahrani MJ. Wernicke's encephalopathy following hyperemesis gravidarum. Indian J Crit Care Med 2014;18:164-6.

4 Chamorro AJ, Rosón-Hernández B, Medina-García JA et al. Differences between alcoholic and nonalcoholic patients with Wernicke encephalopathy: A multicenter observational study. Mayo Clin Proc 2017:92:899.

5 Busani S, Bonvecchio C, Gaspari A et al. Wernicke's encephalopathy in a malnourished surgical patient: a difficult diagnosis. BMC Res Notes 2014;7:718.

6 Zuccoli G, Pipitone N. Neuroimaging findings in acute Wernicke's encephalopathy: review of the literature. AJR Am J Roentgenol 2009:192:501.

7 Ballabio M, Poshyachinda M, Ekins RP. Pregnancy-induced changes in thyroid function: role of human chorionic gonadotropin as putative regulator of maternal thyroid. J Clin Endocrinol 1991;73:824-31.

8 Goodwin TM, Montoro M, Mestman JH, Pekary AE, Hershman JM. The role of chorionic gonadotropin in transient hyperthyroidism of hyperemesis gravidarum. J Clin Endocrinol 1992;75:1333-7.

Address for correspondence: Dr Chin Voon Tong, Jalan Mufti Haji Khalil, 75400 Malacca, Malaysia.

Email: tchinvoon@yahoo.com 